\title{
Controllability and Optimal Strokes for N-link Micro-swimmer
}

\author{
L. GIRALdi ${ }^{1}$, P. MARTINON ${ }^{1}$ AND M. ZOPPELLO ${ }^{3}$ \\ ${ }^{1}$ CMAP, Ecole Polytechnique, 91128 Palaiseau Cedex, France. \\ ${ }^{3}$ Università degli Studi di Padova, Padova, Italy.
}

March 11, 2013

\begin{abstract}
In this paper we focus on the $N$-link swimmer, a generalization of the classical Purcell swimmer [18, that was introduced in [1. We use the simplification of the Resistive Force Theory to derive the equation of motion for the swimmer in a fluid with a low Reynolds number, see for instance [12. We prove that the swimmer is controllable in the whole plane when it is composed by more than 3 sticks and for almost every set of stick lengths. As a direct result, we show that there exists an optimal swimming strategy which leads to minimize the time to reach a desired configuration. Numerical experiments on the case of $N=3$ (Purcell swimmer) suggest that the optimal strategy is periodic, i.e. composed of a sequence of identical strokes. Our results indicate that this candidate for an optimal stroke indeed gives abetter speed than the classical Purcell stroke.
\end{abstract}




\section{Contents}

1 Introduction 2

1.1 Locomotion at low Reynolds Number. . . . . . . . . . . . . . . . 2

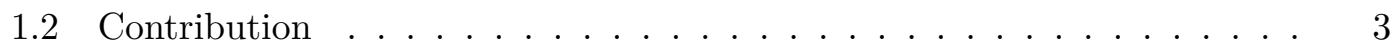

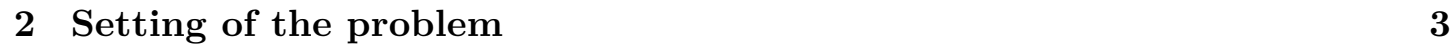

2.1 The $N$-link swimmer . . . . . . . . . . . . . . . . . . . 3

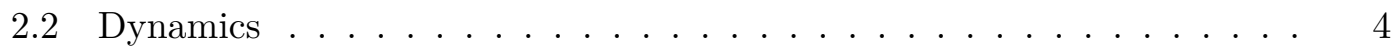

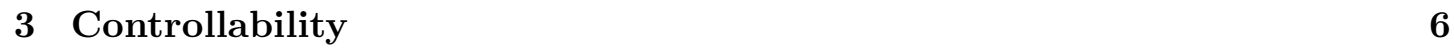

3.1 Classical results in geometric control . . . . . . . . . . . . . . . 6

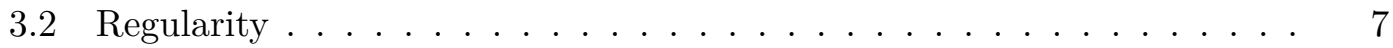

3.3 Controllability of the Purcell Swimmer $(N=3)$. . . . . . . . . . 8

3.4 Controllability of the $N$-link swimmer $\ldots \ldots \ldots$. . . . . . . . 9

\begin{tabular}{|lll}
4 & Minimum time optimal problem for the $N$-link swimmer & 10
\end{tabular}

4.1 Optimal Time Control Problem Statement. . . . . . . . . . . . . . . . . 10

4.2 Optimization Strategy $\ldots \ldots \ldots \ldots \ldots$. . . . . . . . . . . . . 11

$5 \quad$ Numerical simulation for the Purcell's swimmer $(\mathrm{N}=3)$

5.1 The classical Purcell stroke $\ldots \ldots \ldots \ldots$. . . . . . . . . . . . . 13

5.2 Comparison of the optimal stroke with the classical Purcell stroke . . . 14

\begin{tabular}{lll}
\hline 6 & Conclusions & 17
\end{tabular}

\section{Introduction}

\subsection{Locomotion at low Reynolds Number}

Swimming at a micro scale is a subject of growing interest. A better understanding of the swimmer motion can lead to many useful applications in several fields such as medicine or micro and nano technology. The world of low Reynolds Number is inhabited by the majority of the micro organisms, for this reason the study of their swimming strategy is attracting increasing attention in the recent literature (see for instance [14] for an extensive list of references). One of the pioneering works is probably the one by Taylor in 1951 (see [20]) who introduces a model of swimmer as an infinite sheet in the form of a sinusoidal traveling wave. In this paper, Taylor presents a mathematical setting for the problem of self-propulsion of this thin undulating filament. Later in 1977, Purcell proved in [18] that the swimming strategies must change the shape of the swimmer in a non-reciprocal way, in order to permit a displacement through the fluid. In the same paper, he introduced a 3-link swimmer model, known as the "Purcell swimmer", along with a stroke that allows it to move. More recently, several works have studied in more detail the physical characteristic of the Purcell swimmer as a toy model, see for instance [19], 6], 11, [17]. Another crucial development for our analysis is the recent emergence of 
the connection between swimming and Control Theory (see for instance [16], 3], [4], 8], [15], [2]). One of the difficulties is the study of the swimmer-fluid coupling which leads to derive the dynamics of the swimmer. At a micro scale, the non local hydrodynamic forces exerted by the fluid on the swimmer can be approximated with local drag forces depending linearly on the velocity of each point (see [12], [10]). This technique called Resistive Force Theory provides a simplified dynamics of the micro swimmer, that gives results in good agreement with those obtained by the full hydrodynamic, see [1], [10]. We use here the same approach than [1] to derive the dynamics of the $N$-link swimmer.

\subsection{Contribution}

In this paper, we present a controllability result for the $N$-link swimmer, and a new optimal stroke for displacement in minimum time. First, we prove by geometric control techniques that for $N \geqslant 3$ sticks, the $N$-link swimmer is capable to reach any configuration in the plane. More precisely, we show that for almost any swimmer (i.e. for almost every set of stick lengths) and for any initial configuration, the swimmer can reach any shape and position. The global controllability result proved here shows the existence of a suitable shape deformation which steers the swimmer to the desired final state. As a direct consequence, we show that the optimal swimming problem, that is to minimize the time to reach a given configuration, is well posed. Therefore, there exists an optimal strategy which leads to the final position and configuration in minimum time. Finally, we perform some numerical simulations for the Purcell swimmer $(N=3)$, without any assumptions on the structure of the optimal strategy. Our results suggest that the optimal swimming motion is indeed periodic, and we show that the stroke we obtain gives a better speed than the Purcell one.

\section{Setting of the problem}

In this section, we recall the $N$-link swimmer introduced in [1], and present its dynamics as a system of three ODEs. The system is linear with respect to the rate of deformation, and has no drift.

\subsection{The $N$-link swimmer}

The swimmer consists of $N \in \mathbf{N}$ rigid links with joints at their ends, see Fig. 1 . Movement is expressed in the laboratory-frame, defined by the vectors $\left(\mathbf{e}_{x}, \mathbf{e}_{y}\right)$. We set $\mathbf{e}_{z}:=\mathbf{e}_{x} \times \mathbf{e}_{y}$. The $i$-th link is the segment with end points $\mathbf{x}_{i}$ and $\mathbf{x}_{i+1}$. We note its length $L_{i}>0$ and $\theta_{i}$ its angle with the horizontal $x$-axis. We define by $\mathbf{x}_{i}:=\left(x_{i}, y_{i}\right)$ $(i=1, \cdots, N)$ the coordinates of the first end of each link. Note that, for $i \in\{2 \cdots N\}$, the coordinates $\mathbf{x}_{i}$ can be expressed as a function of $\mathbf{x}_{1}, \theta_{k}$ and $L_{k}$, with $k \in\{1 \cdots i-1\}$ :

$$
\mathbf{x}_{i}:=\mathbf{x}_{1}+\sum_{k=1}^{i-1} L_{k}\left(\begin{array}{c}
\cos \left(\theta_{k}\right) \\
\sin \left(\theta_{k}\right)
\end{array}\right) .
$$

The swimmer is described by two sets of variables: 
- the state variables which specify the position and the orientation of the first link, associated with the triplet $\left(\mathbf{x}_{1}=\left(x_{1}, y_{1}\right), \theta_{1}\right)$.

- the shape variables which describe the relative orientations between successive links. For each link with $i \in[2, \cdots, N]$, we note $\alpha_{i}=\theta_{i}-\theta_{i-1}$ the angle relative to the preceding one. In the following, the vector $\left(\alpha_{2}, \ldots, \alpha_{N}\right)$ represents the shape of the swimmer.

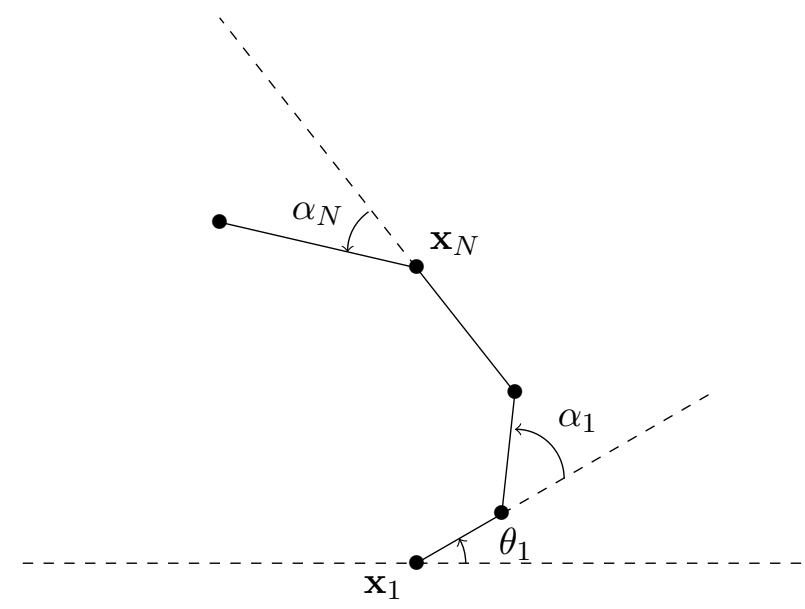

Figure 1: Coordinates for the N-link swimmer.

\subsection{Dynamics}

The dynamics for this swimmer was already described in [1]. We recall in this section the main steps to obtain the equations of motion.

The dynamics of the swimmer stems from Newton laws, in which inertia is neglected. These read

$$
\left\{\begin{array}{l}
\mathbf{F}=0 \\
\mathbf{e}_{z} \cdot \mathbf{T}_{\mathbf{x}_{1}}=0
\end{array}\right.
$$

where $\mathbf{F}$ is the total force exerted on the swimmer by the fluid and $\mathbf{T}_{\mathbf{x}_{1}}$ is the corresponding total torque computed with respect to the point $\mathbf{x}_{1}$.

To couple the fluid and the swimmer, we use the local drag approximation of Resistive Force Theory. We denote by $s$ the arc length coordinate on the $i$-th link $\left(0 \leq s \leq L_{i}\right)$ and by $\mathbf{v}_{i}(s)$ the velocity of the corresponding point. We also introduce the unit vectors in the directions parallel and perpendicular to the $i$-th link

$\mathbf{e}_{i}=\left(\begin{array}{c}\cos \left(\theta_{i}\right) \\ \sin \left(\theta_{i}\right)\end{array}\right)$ and $\mathbf{e}_{i}^{\perp}=\left(\begin{array}{c}-\sin \left(\theta_{i}\right) \\ \cos \left(\theta_{i}\right)\end{array}\right)$ 
and write $\mathbf{x}_{i}(s)=\mathbf{x}_{i}+s \mathbf{e}_{i}$. By differentiation, we obtain,

$$
\mathbf{v}_{i}(s)=\dot{\mathbf{x}}_{i}+s \dot{\theta}_{i} \mathbf{e}_{i}^{\perp} .
$$

The density of the force $\mathbf{f}_{i}$ acting on the $i$-th segment is assumed to depend linearly on the velocity. It is defined by

$$
\mathbf{f}_{i}(s):=-\xi\left(\mathbf{v}_{i}(s) \cdot \mathbf{e}_{i}\right) \mathbf{e}_{i}-\eta\left(\mathbf{v}_{i}(s) \cdot \mathbf{e}_{i}^{\perp}\right) \mathbf{e}_{i}^{\perp},
$$

where $\xi$ and $\eta$ are respectively the drag coefficients in the directions of $\mathbf{e}_{i}$ and $\mathbf{e}_{i}^{\perp}$. We thus obtain

$$
\left\{\begin{array}{l}
\mathbf{F}=\sum_{i=1}^{N} \int_{0}^{L_{i}} \mathbf{f}_{i}(s) d s \\
\mathbf{e}_{z} \cdot \mathbf{T}_{\mathbf{x}_{1}}=\mathbf{e}_{z} \cdot \sum_{i=1}^{N} \int_{0}^{L_{i}}\left(\mathbf{x}_{i}(s)-\mathbf{x}_{1}\right) \times \mathbf{f}_{i}(s) d s .
\end{array}\right.
$$

Using (3) and (4) into (5), the total force $\mathbf{F}$ can be expressed as

$$
-\sum_{i=1}^{N} L_{i} \xi\left(\dot{\mathbf{x}}_{i} \cdot \mathbf{e}_{i}\right) \mathbf{e}_{i}+\left(L_{i} \eta\left(\dot{\mathbf{x}}_{i} \cdot \mathbf{e}_{i}^{\perp}\right)+\frac{L_{i}^{2}}{2} \eta \dot{\theta}_{i}\right) \mathbf{e}_{i}^{\perp},
$$

and and torque $\mathbf{e}_{z} \cdot \mathbf{T}_{\mathbf{x}_{1}}$ as

$$
\begin{aligned}
-\sum_{i=1}^{N} & L_{i} \eta\left(\dot{\mathbf{x}}_{i} \cdot \mathbf{e}_{i}^{\perp}\right)\left(\mathbf{x}_{i}-\mathbf{x}_{1}\right) \times \mathbf{e}_{i}^{\perp}+ \\
& L_{i} \xi\left(\dot{\mathbf{x}}_{i} \cdot \mathbf{e}_{i}\right)\left(\mathbf{x}_{i}-\mathbf{x}_{1}\right) \times \mathbf{e}_{i}+ \\
& \frac{L_{i}^{2}}{2} \eta \dot{\theta}_{i} \quad\left(\mathbf{x}_{i}-\mathbf{x}_{1}\right) \times \mathbf{e}_{i}^{\perp}+ \\
& \frac{L_{i}^{2}}{2} \eta\left(\dot{\mathbf{x}}_{i} \cdot \mathbf{e}_{i}^{\perp}\right)+\frac{L_{i}^{3}}{3} \eta \dot{\theta}_{i} .
\end{aligned}
$$

Moreover, differentiating (1) gives

$$
\dot{\mathbf{x}}_{i}=\dot{\mathbf{x}}_{1}+\sum_{k=1}^{i-1} L_{k} \dot{\theta}_{k} \mathbf{e}_{k}^{\perp},
$$

which is linear in $\dot{\mathbf{x}}_{1}$ and $\left(\dot{\theta}_{k}\right)_{1 \leq k \leq N}$.

The angles $\left(\dot{\alpha}_{k}\right)_{2 \leq k \leq N}$ are a linear combinaison of $\left(\dot{\theta}_{k}\right)_{2 \leq k \leq N}$, thus formulas (6) and (7) are linear in $\dot{\mathbf{x}}_{1}, \dot{\theta}_{1}$ and $\left(\dot{\alpha}_{k}\right)_{2 \leq k \leq N}$.

Writing the system 5 in a matricial form we obtain

$$
\mathbf{A}\left(\theta_{1}, \alpha_{2}, \cdots, \alpha_{N}\right) \cdot\left(\begin{array}{c}
x_{1} \\
y_{1} \\
\theta_{1}
\end{array}\right)-\mathbf{B}\left(\theta_{1}, \alpha_{2}, \cdots, \alpha_{N}\right) \cdot\left(\begin{array}{c}
\alpha_{2} \\
\vdots \\
\alpha_{N}
\end{array}\right)=0
$$


where the matrix $\mathbf{A}\left(\theta_{1}, \alpha_{2}, \cdots, \alpha_{N}\right)$ is known as "Grand Resistance Matrix", and $\mathbf{B}\left(\theta_{1}, \alpha_{2}, \cdots, \alpha_{N}\right)$ is the linear map associated to the coefficients describing the shape of the swimmer [1].

By inverting $\mathbf{A}$, we get the existence of the family of vector fields $\left\{\tilde{\mathbf{g}}_{i}\left(\theta_{1}, \alpha_{2}, \cdots, \alpha_{N}\right)\right\}_{i=1, \cdots, N-1}$ defined on $[0,2 \pi]^{N}$ by $\tilde{\mathbf{g}}_{i}:=\mathbf{A}^{-1} \mathbf{B}$. Then the dynamics of the swimmer reads

$$
\left(\begin{array}{c}
\dot{\alpha}_{2} \\
\vdots \\
\dot{\alpha}_{N} \\
\dot{\mathbf{x}}_{1} \\
\dot{\theta}_{1}
\end{array}\right)=\sum_{i=1}^{N-1}\left(\begin{array}{c}
\mathbf{b}_{i} \\
\tilde{\mathbf{g}}_{i}\left(\theta_{1}, \alpha_{2}, \cdots, \alpha_{N}\right)
\end{array}\right) \dot{\alpha}_{i+1} .
$$

where $\mathbf{b}_{i}$ is the $i$-th vector of the canonical basis of $\mathbf{R}^{N-1}$.

\section{Controllability}

This Section is devoted to the controllability result of the $N$-link swimmer. Namely, we prove that there exist control functions which allow the swimmer to move everywhere in the plane.

Theorem 3.1 Consider the $N$-link swimmer described in Section 2 evolving in the space $\mathbf{R}^{2}$. Then for almost every lengths of the sticks $\left(L_{i}\right)_{i=1, \cdots, N}$ and for any initial configuration

$\left(\mathbf{x}_{1}^{i}, \theta_{1}^{i}, \alpha_{2}^{i}, \cdots, \alpha_{N}^{i}\right) \in \mathbf{R}^{2} \times[0,2 \pi]^{N}$, any final configuration $\left(\mathbf{x}_{1}^{f}, \theta_{1}^{f}, \alpha_{2}^{f}, \cdots, \alpha_{N}^{f}\right)$ and any final time $T>0$, there exists a shape function $\left(\alpha_{2}, \cdots, \alpha_{N}\right) \in \mathcal{W}^{1, \infty}([0, T])$, satisfying $\left(\alpha_{2}, \cdots, \alpha_{N}\right)(0)=\left(\alpha_{2}^{i}, \cdots, \alpha_{N}^{i}\right)$ and $\left(\alpha_{2}, \cdots, \alpha_{N}\right)(T)=\left(\alpha_{2}^{f}, \cdots, \alpha_{N}^{f}\right)$ and such that if the self-propelled swimmer starts in position $\left(\mathrm{x}_{1}^{i}, \theta_{1}^{i}\right)$ with the shape $\left(\alpha_{2}^{i}, \cdots, \alpha_{N}^{i}\right)$ at time $t=0$, it ends at position $\left(\mathbf{x}_{1}^{f}, \theta_{1}^{f}\right)$ and shape $\left(\alpha_{2}^{f}, \cdots, \alpha_{N}^{f}\right)$ at time $t=T$ by changing its shape along $\left(\alpha_{2}, \cdots, \alpha_{N}\right)(t)$.

Proof: The proof of the theorem is divided into three steps. First, we deal with the analyticity of the dynamics vector fields. Then, we prove the controllability of the Purcell 3-link swimmer, exploiting the Chow theorem and the Orbit theorem. Finally, we generalize the result to the $N$-link swimmer. We start by recalling some classical results used in the proof.

\subsection{Classical results in geometric control}

Theorem 3.2 (Chow (see [9])) Let $m, n \in \mathbf{N}$ and let $\left(f_{i}\right)_{i=1, n}$ be $\mathcal{C}^{\infty}$ vector fields on $\mathbf{R}^{n}$. Consider the control system, of state trajectory $\mathbf{q}$,

$$
\dot{q}=\sum_{i=1}^{m} u_{i} f_{i}(\mathbf{q})
$$


with input function $\mathbf{u}=\left(u_{i}\right)_{i=1, m} \in L^{\infty}\left(\left[0,+\infty\left[, \mathbf{B}_{\mathbf{R}^{n}}(0, r)\right)\right.\right.$ for some $r>0$.

Let $\mathcal{O}$ an open and connected set of $\mathbf{R}^{n}$ and assume that

$$
\mathbf{L} i e_{\mathbf{q}}\left(f_{1}, \ldots f_{m}\right)=\mathbf{R}^{n} \mathbf{q} \in \mathcal{O} .
$$

Then the system (11) is controllable, i.e., for every $\mathbf{q}_{0}, \mathbf{q}_{1}$ in $\mathcal{O}$ and for every $T>0$ exists $\mathbf{u} \in L^{\infty}\left((0, T), \mathbf{B}_{\mathbf{R}^{n}}(0, r)\right)$ such that $\mathbf{q}(0)=\mathbf{q}_{0}$ and $\mathbf{q}(T)=\mathbf{q}_{1}$ and $\mathbf{q}(t) \in \mathcal{O}$ for every $t \in[0, T]$.

If the vector fields are analytic, we can apply the Orbit Theorem to extend the dimension property of the Lie algebra defined by the dynamics vector fields on the whole orbit.

Theorem 3.3 (Orbit (see [13]) Let $\mathcal{M}$ be an analytic manifold, and $\mathcal{F}$ a family of analytic vector fields on $\mathcal{M}$. Then

a) each orbit of $\mathcal{F}$ is an analytic submanifold of $\mathcal{M}$, and

b) if $N$ is an orbit of $\mathcal{F}$, then the tangent space of $\mathcal{N}$ at $x$ is given by $\operatorname{Lie}_{x}(\mathcal{F})$. In particular the dimension of $\operatorname{Lie}_{x}(\mathcal{F})$ is constant as $x$ varies on $\mathcal{N}$.

In our case, the manifold in which the state and the shape of the swimmer evolve is defined by $\mathcal{M}:=[0,2 \pi]^{N-1} \times \mathbf{R}^{2} \times[0,2 \pi]$ The vector fields of the dynamics are denoted by

$$
\mathbf{g}_{i}\left(\theta_{1}, \alpha_{2}, \cdots, \alpha_{N}\right):=\left(\begin{array}{c}
\mathbf{b}_{i} \\
\tilde{\mathbf{g}_{i}}\left(\theta_{1}, \alpha_{2}, \cdots, \alpha_{N}\right)
\end{array}\right) .
$$

We say that the Lie algebra of the family of vector fields $\left\{\mathbf{g}_{i}\right\}_{i=1, \cdots, N-1}$ is fully generated at the point $\mathbf{q}=\left(\alpha_{2}, \cdots, \alpha_{N}, x_{1}, y_{1}, \theta_{1}\right) \in \mathcal{M}$ if the tangent space of the manifold, $T_{\mathbf{q}} \mathcal{M}$, is equal to the Lie algebra $\left.\operatorname{Lie}\left(\left(\mathbf{g}_{i}\right)\right)_{i=1, \cdots, N-1}\right)(\mathbf{q})$.

\subsection{Regularity}

The first step is to prove that the vector fields of the motion equation of the swimmer are analytic on $\mathcal{M}$.

As a direct consequence of (6) and (7), the linear maps $\mathbf{A}$ and $\mathbf{B}$ belong to the set of matrices whose entries are analytic functions on $[0,2 \pi]^{N}$. The family of vector $\left(\tilde{\mathbf{g}}_{i}\left(\theta_{1}, \alpha_{2}, \cdots, \alpha_{N}\right)\right)_{i=1, \cdots, N-1}$ is obtained by the multiplication of $\mathbf{A}^{-1}$ by $\mathbf{B}$. Since the coefficients of $\mathbf{A}^{-1}$ are obtained by multiplication and division of those of $\mathbf{A}$, and because the determinant of $\mathbf{A}$ is never null, the entries of inverse matrix $\mathbf{A}^{-1}$ remain analytic functions on $[0,2 \pi]^{N}$. Thus, the family of vector fields $\left(\tilde{\mathbf{g}}_{i}\right)_{i=1, \cdots, N}$ are analytic on $[0,2 \pi]^{N}$. 


\subsection{Controllability of the Purcell Swimmer $(N=3)$}

Now we prove the controllability of the Purcell's swimmer. By replacing $N=3$ in 10 , the Purcell's dynamics reads

$$
\left(\begin{array}{c}
\dot{\alpha}_{2} \\
\dot{\alpha}_{3} \\
\dot{x}_{1} \\
\dot{y}_{1} \\
\dot{\theta}_{1}
\end{array}\right)=\mathbf{g}_{1}\left(\theta_{1}, \alpha_{2}, \alpha_{3}\right) \dot{\alpha}_{2}+\mathbf{g}_{2}\left(\theta_{1}, \alpha_{2}, \alpha_{3}\right) \dot{\alpha}_{3} .
$$

We now express the Lie algebra of the vector fields $\mathbf{g}_{1}$ and $\mathbf{g}_{2}$ for any $\theta_{1} \in[0,2 \pi]$ at the point $\left(\alpha_{2}, \alpha_{3}\right)=(0,0)$, for a swimmer whose sticks have the length $L_{1}=L_{3}=L$ and $L_{2}=2 L$ where $L>0$.

The two vectors $\mathbf{g}_{1}\left(\theta_{1}, 0,0\right)$ and $\mathbf{g}_{2}\left(\theta_{1}, 0,0\right)$ are

$$
\mathbf{g}_{1}\left(\theta_{1}, 0,0\right)=\left(\begin{array}{c}
1 \\
0 \\
\frac{9 L \sin \left(\theta_{1}\right)}{64} \\
-\frac{9 L \cos \left(\theta_{1}\right)}{64} \\
\frac{27}{32}
\end{array}\right), \quad \mathbf{g}_{2}\left(\theta_{1}, 0,0\right)=\left(\begin{array}{c}
0 \\
1 \\
-\frac{7 L \sin \left(\theta_{1}\right)}{64} \\
\frac{7 L \cos \left(\theta_{1}\right)}{64} \\
-\frac{5}{32}
\end{array}\right) .
$$

Then, the iterated Lie brackets are equals to

$$
\left[\mathbf{g}_{1}, \mathbf{g}_{2}\right]\left(\theta_{1}, 0,0\right)=\left(\begin{array}{c}
0 \\
0 \\
\frac{7 L(\eta-\xi) \cos \left(\theta_{1}\right)}{128 \xi} \\
\frac{7 L(\eta-\xi) \sin \left(\theta_{1}\right)}{128 \xi} \\
0
\end{array}\right)
$$




$$
\left[\mathbf{g}_{1},\left[\mathbf{g}_{1}, \mathbf{g}_{2}\right]\right]\left(\theta_{1}, 0,0\right)=\left(\begin{array}{c}
0 \\
0 \\
-\frac{L\left(126 \eta^{2}+31 \xi \eta-76 \xi^{2}\right) \sin \left(\theta_{1}\right)}{4096 \eta \xi} \\
\frac{L\left(126 \eta^{2}+31 \xi \eta-76 \xi^{2}\right) \cos \left(\theta_{1}\right)}{4096 \eta \xi} \\
-\frac{3\left(9 \eta^{2}-4 \xi \eta+4 \xi^{2}\right)}{2048 \eta \xi}
\end{array}\right),
$$

The determinant of the matrix whose columns are the previous vector fields is equal to

$$
\begin{aligned}
& \left|\left(\mathbf{g}_{1} \mathbf{g}_{2}\left[\mathbf{g}_{1}, \mathbf{g}_{2}\right]\left[\mathbf{g}_{1},\left[\mathbf{g}_{1}, \mathbf{g}_{2}\right]\right]\left[\mathbf{g}_{2},\left[\mathbf{g}_{1}, \mathbf{g}_{2}\right]\right]\right)\left(\theta_{1}, 0,0\right)\right| \\
& \quad=\frac{21 L^{2}(\eta-\xi)^{2}(45 \eta+112 \xi)\left(9 \eta^{2}-4 \eta \xi+4 \xi^{2}\right)}{536870912 \eta^{2} \xi^{3}}
\end{aligned}
$$

Since the two drag coefficients $\xi$ and $\eta$, are supposed positive, this determinant is never null exept for isotropic coefficients $(\xi=\eta)$. If we assume the drag coefficients to be equal, physically means that the sticks are subjected to the same drag force in both paralel and orthogonal dirctions, so they would not be sticks but spheres. Thus this case is not physically acceptable.

Thus for any $\theta_{1} \in[0,2 \pi]$, the Lie algebra of the vector fields $\mathbf{g}_{1}$ and $\mathbf{g}_{2}$ is fully generated at the point $\left(\theta_{1}, \alpha_{2}, \alpha_{3}\right)=\left(\theta_{1}, 0,0\right)$. Remark that any point $\left(\alpha_{2}, \alpha_{3}, \mathbf{x}_{1}, \theta_{1}\right) \in$ $[0,2 \pi]^{2} \times \mathbf{R}^{2} \times[0,2 \pi]$ belongs to the orbit of the point $\left(0,0, \mathbf{x}_{1}, \theta_{1}\right)$. Since the vector fields are analytic, Orbit Theorem 3.3 guarantees that the Lie algebra of $\mathbf{g}_{1}$ and $\mathbf{g}_{2}$ is fully generated everywhere in the manifold $[0,2 \pi]^{2} \times \mathbf{R}^{2} \times[0,2 \pi]$.

To conclude, by Chow Theorem 3.2 we get the controllability of the Purcell's swimmer whose sticks have same length.

\subsection{Controllability of the $N$-link swimmer}

The third step is to generalize the previous controllability result to the N-link swimmer. 
The dynamics of this swimmer is described by the ODE $(10)$. By construction, the family of vector fields $\mathbf{g}_{i}$ generates the tangent space of the manifolds $[0,2 \pi]^{N-1}$,

$$
\operatorname{Span}\left(\mathbf{g}_{1}, \cdots, \mathbf{g}_{N-1}\right)=\mathbf{R}^{N-1} .
$$

The two vector fields $\mathbf{g}_{1}$ and $\mathbf{g}_{2}$ are related to the Purcell's one defined in (12): we add $N-2$ rows of zeroes, take sticks of null length $L_{i}=0$ for $4 \leq i \leq N-1$, while keeping the three sticks $L_{1}=L_{3}=L$ and $L_{2}=2 L$ unchanged.

In this case, for any $\left(\mathbf{x}_{1}, \theta_{1}\right) \in \mathbf{R}^{2} \times[0,2 \pi]$ Subsection 3.3 shows that the vectors $\mathbf{g}_{1}\left(\theta_{1}, 0, \cdots 0\right), \mathbf{g}_{2}\left(\theta_{1}, 0, \cdots 0\right)$ and their iterated Lie brackets $\left[\mathbf{g}_{1}, \mathbf{g}_{2}\right]\left(\theta_{1}, 0, \cdots 0\right)$, $\left[\mathbf{g}_{1},\left[\mathbf{g}_{1}, \mathbf{g}_{2}\right]\right]\left(\theta_{1}, 0, \cdots 0\right)$, and $\left[\mathbf{g}_{2},\left[\mathbf{g}_{1}, \mathbf{g}_{2}\right]\right]\left(\theta_{1}, 0, \cdots 0\right)$ are linearly independent.

Therefore, the Lie algebra of the family $\left(\mathbf{g}_{i}\right)_{i=1, \cdots, N-1}$ at the point $\left(\theta_{1}, 0, \cdots, 0\right)$ is equal to the tangent space $T_{\left(0, \cdots, 0, \mathbf{x}_{1}, \theta_{1}\right)} \mathcal{M}$.

Then, by analyticity of the vector fields $\mathbf{g}_{i}$, Orbit Theorem 3.3 states that the Lie algebra is fully generated everywhere for a swimmer whose the length of sticks verify $L_{1}=L_{3}=L, L_{2}=2 L$ and $L_{i}=0$, for $4 \leq i \leq N-1$.

Notice that the vector fields of the motion equation depend analytically also on the sticks length $L_{i}, i=1, \cdots, N$. We define by $D^{(0, \cdots, 0)}$, the function which associates to the $N$-uplet of the stick lengths the determinant of the vectors $\mathbf{g}_{1}(0, \cdots, 0), \cdots, \mathbf{g}_{N-1}(0, \cdots, 0)$ and their iterated Lie brakets at $(0, \cdots, 0)$.

Since the dependance on $L_{i}$ of vector fields $\mathbf{g}_{i}$ is analytic, we get the analyticity of the function $D^{(0, \cdots, 0)}$. Thus for any $L>0$, the value of $D^{(0, \cdots, 0)}$ at the point $(L, 2 L, L, 0 \cdots 0)$ is not null. Then, by analyticity it remains non null almost everywhere in $\mathbf{R}^{N}$. Therefore, we obtain that the Lie algebra of a full rank for almost every swimmer.

Finally, by using Chow Theorem 3.2 , we get the controllability stated in the Theorem 3.1

\section{Minimum time optimal problem for the $N$-link swimmer}

This Section describes the minimum time optimal control problem for the $N$-link swimmer. The problem is defined in 4.1, and is well defined, from the controllability result proven in 3 . Then in 4.2 we present the optimization strategy we used to find a solution to this optimal control problem.

\subsection{Optimal Time Control Problem Statement}

For any time $t>0$, let us denote the state of the swimmer by $\mathbf{z}(t):=\left(\alpha_{2}, \cdots, \alpha_{N}, \mathbf{x}_{1}, \theta_{1}\right)(t)^{T}$, the control function by $\mathbf{u}(t):=\left(\dot{\alpha}_{2}, \cdots, \dot{\alpha}_{N}\right)(t)$ and the dynamics by $\mathbf{f}(\mathbf{z}(t), \mathbf{u}(t))=$ $\sum_{i=1}^{N-1} \mathbf{g}_{i}(\mathbf{z}(t)) \dot{\alpha}_{i+1}(t)$.

In the following we assume that the swimmer starts at the initial configuration $\mathbf{z}^{i}$ and 
we set a final state $\mathbf{z}^{f}$. We want to find an optimal swimming strategy which minimizes the time to reach the final configuration, i.e.,

$$
(O C P)\left\{\begin{array}{l}
\inf T, \\
\dot{\mathbf{z}}(t)=f(\mathbf{z}(t), \mathbf{u}(t)), \forall t \in[0, T], \\
\mathbf{u}(t) \in \mathbf{U}:=[-1,1]^{N}, \forall t \in[0, T], \\
\mathbf{z}(0)=\mathbf{z}^{i}, \\
\mathbf{z}(T)=\mathbf{z}^{f} .
\end{array}\right.
$$

By rescaling the dynamics 10 , from the controllability result 3.1 the following statement holds.

Corollary 4.1 Consider the $N$-link swimmer described in Section 2 evolving in $\mathbf{R}^{2}$. Then for almost all lengths $\left(L_{i}\right)_{i=1, \cdots, N}$ and for any initial configuration $\left(\mathbf{x}_{1}^{i}, \theta_{1}^{i}, \alpha_{2}^{i}, \cdots, \alpha_{N}^{i}\right)$, any final configuration $\left(\mathbf{x}_{1}^{f}, \theta_{1}^{f}, \alpha_{2}^{f}, \cdots, \alpha_{N}^{f}\right)$ and any final time $T>0$, there exists a function $\left(\alpha_{2}, \cdots, \alpha_{N}\right) \in \mathcal{W}^{1, \infty}([0, T])$ such that $\left(\dot{\alpha}_{2}, \cdots, \dot{\alpha}_{N}\right) \in \mathbf{U}$, satisfying $\left(\alpha_{2}, \cdots, \alpha_{N}\right)(0)=$ $\left(\alpha_{2}^{i}, \cdots, \alpha_{N}^{i}\right)$ and $\left(\alpha_{2}, \cdots, \alpha_{N}\right)(T)=\left(\alpha_{2}^{f}, \cdots, \alpha_{N}^{f}\right)$ and such that if the self-propelled swimmer starts in position $\mathbf{x}_{1}^{i}, \theta_{1}^{i}$ with the shape $\alpha_{2}^{i}, \cdots, \alpha_{N}^{i}$ at time $t=0$, it ends at position $\left(\mathbf{x}_{1}^{f}, \theta_{1}^{f}\right)$ and shape $\alpha_{2}^{f}, \cdots, \alpha_{N}^{f}$ at time $t=T$ by changing its shape along $\left(\alpha_{2}, \cdots, \alpha_{N}\right)(t)$.

This corollary means that the displacement of the swimmer does not depend of the speed of the shape changes of the swimmer. By applying Filippov-Cesary Theorem (as stated in [21]), there exist a minimal time such that the constraints are satisfied, and the optimal problem reads

$$
(O C P)\left\{\begin{array}{l}
\min T \\
\dot{\mathbf{z}}(t)=f(\mathbf{z}(t), \mathbf{u}(t)), \forall t \in[0, T] \\
\mathbf{u}(t) \in \mathbf{U}:=[-1,1]^{N}, \forall t \in[0, T], \\
\mathbf{z}(0)=\mathbf{z}^{i} \\
\mathbf{z}(T)=\mathbf{z}^{f}
\end{array}\right.
$$

\subsection{Optimization Strategy}

In order to solve this optimal control problem, we use a so-called direct approach. The direct approach transforms the infinite dimensional optimal control problem $(O C P)$ into a finite dimensional optimization problem $(N L P)$. This is done by a discretization in time applied to the state and control variables, as well as the dynamics equation. These methods are usually less precise than indirect methods based on Pontryagin's Maximum Principle, but more robust with respect to the initialization. Also, they are more straightforward to apply, hence they are widely used in industrial applications. 
Summary of the time discretization:

$$
\begin{array}{ll}
t \in[0, T] & \rightarrow\left\{t_{0}=0, \ldots, t_{N}=T\right\} \\
z(\cdot), u(\cdot) & \rightarrow X=\left\{z_{0}, \ldots, z_{N}, u_{0}, \ldots, u_{N-1}, T\right\} \\
\hline \text { Criterion } & \rightarrow \min T \\
\text { Dynamics } & \rightarrow(\text { ex: Euler }) z_{i+i}=z_{i}+h f\left(z_{i}, u_{i}\right) \\
\text { Adm. Cont. } & \rightarrow-1 \leqslant u_{i} \leqslant 1 \\
\text { Bnd. Cond. } & \rightarrow \Phi\left(z_{0}, z_{N}\right)=0
\end{array}
$$

We therefore obtain a nonlinear programming problem on the discretized state and control variables

$$
(N L P)\left\{\begin{array}{l}
\min F(z)=T \\
L B \leqslant C(z) \leqslant U B
\end{array}\right.
$$

All tests were run using the software Bocor ${ }^{1}([7])$. The discretized nonlinear optimization problem is solved by the well-known solver IPOPT [22] with MUMPs [5], while the derivatives are computed by sparse automatic differentiation with ADOL-C 23 and ColPACK [11. In the numerical experiments, we used a Midpoint (implicit 2nd order) discretization with 1000 time steps. Execution times on a Xeon $3.2 \mathrm{GHz}$ CPU were a few minutes.

\section{Numerical simulation for the Purcell's swimmer $(\mathrm{N}=3)$}

In this Section, we present the numerical simulations associated with the problem (15) in the case of $N=3$ sticks (Purcell's swimmer). We observe that while we did not make any assumptions on the structure of the optimal trajectory, the solution given by the direct solver Bocop shows a periodic structure. We extract a stroke from these solutions, and check that we obtain a better displacement better than the one of Purcell ([18, [6]).

In the rest of the paper, we reformulate the system in order to match the state variables used in the literature for the Purcell swimmer [6]. Following [6], we take the sticks lengths $L_{1}=L_{3}=1$ and $L_{2}=2$. From now on, the state of the swimmer (see Fig 2 is described by

- the position $\left(x_{2}, y_{2}\right)$ of the center of the second stick, and $\theta_{2}:=\theta_{1}-\alpha_{2}$ the angle between the $\mathrm{x}$-axis and the second stick

- the shape of the swimmer, defined by the two angles $\beta_{1}:=-\alpha_{2}$ and $\beta_{3}:=\alpha_{3}$.

The time derivative of the new variables which describe the swimmer are linear in the previous ones,

$$
\left(\begin{array}{c}
\dot{\beta}_{1} \\
\dot{\beta}_{3} \\
\dot{\mathbf{x}}_{2} \\
\dot{\theta}_{2}
\end{array}\right)=\mathbf{M}\left(\theta_{2}, \beta_{1}\right)\left(\begin{array}{c}
\dot{\alpha}_{2} \\
\dot{\alpha}_{3} \\
\dot{\mathbf{x}}_{1} \\
\dot{\theta}_{1}
\end{array}\right)
$$

\footnotetext{
${ }^{1}$ http://bocop.org
} 


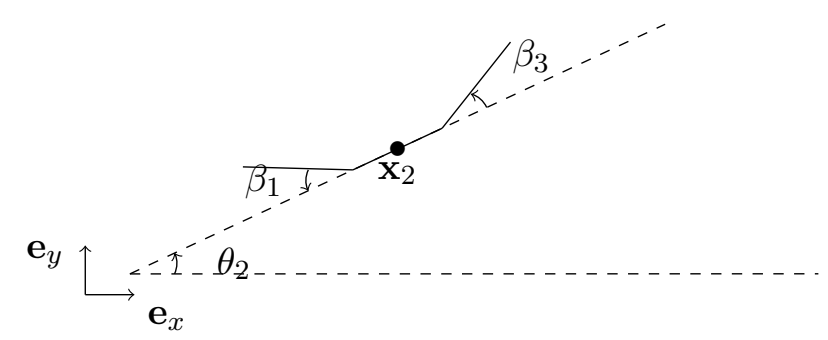

Figure 2: Purcell's 3-link swimmer.

where the matrix $\mathbf{M}\left(\theta_{2}, \beta_{1}\right)$ is defined by,

$$
\mathbf{M}\left(\theta_{2}, \beta_{1}\right)=\left(\begin{array}{ccccc}
-1 & 0 & 0 & 0 & 0 \\
0 & 1 & 0 & 0 & 0 \\
\sin \left(\theta_{2}\right)+\cos \left(\beta_{1}\right) & 0 & 1 & 0 & -\sin \left(\theta_{2}\right) \\
-\cos \left(\beta_{1}\right)-\cos \left(\theta_{2}\right) & 0 & 0 & 1 & \cos \left(\theta_{2}\right) \\
-1 & 0 & 0 & 0 & 1
\end{array}\right) .
$$

As a result, the dynamics 10 reads in this case

$$
\left(\begin{array}{c}
\dot{\beta}_{1} \\
\dot{\beta}_{3} \\
\dot{\mathbf{x}}_{2} \\
\dot{\theta}_{2}
\end{array}\right)=\tilde{\mathbf{f}}_{1}\left(\theta_{2}, \beta_{2}, \beta_{3}\right) \dot{\beta}_{1}+\tilde{\mathbf{f}}_{2}\left(\theta_{2}, \beta_{2}, \beta_{3}\right) \dot{\beta}_{3}
$$

where for $i=1,2$

$$
\tilde{\mathbf{f}}_{i}\left(\theta_{2}, \beta_{1}, \beta_{3}\right)=\mathbf{M}\left(\theta_{2}, \beta_{1},\right) \tilde{\mathbf{g}}_{i}\left(\theta_{1}, \alpha_{2}, \alpha_{3}\right) .
$$

Since the variables which describe the swimmer are the image of the previous one by a one-to-one mapping, it is clear that the controllability result proved in Section 3.3 holds for the ODE (16).

\subsection{The classical Purcell stroke}

The stroke presented by Purcell in [18] is used in the rest to compare the optimal strategy given by our numerical results. Let us denote by $\Delta \theta$, the angular excursion of $\beta_{1}$ and $\beta_{3}$. It means that during the stroke $\beta_{1}$ and $\beta_{3}$ belong to the interval $\left[-\frac{\Delta \theta}{2}, \frac{\Delta \theta}{2}\right]$. Calling $T$ the interval of time in which the swimmer performs the stroke, the Purcell stroke is defined by the following periodic cycle of deformation,

$$
\beta_{1}(t)=\left\{\begin{array}{ll}
\frac{4 \Delta \theta}{T} t-\frac{\Delta \theta}{2} & \text { if } 0 \leq t \leq \frac{T}{4} \\
\frac{\Delta \theta}{2} & \text { if } \frac{T}{4} \leq t \leq \frac{T}{2} \\
-\frac{4 \Delta \theta}{T} t+\frac{5 \Delta \theta}{2} & \text { if } \frac{T}{2} \leq t \leq \frac{3 T}{4} \\
-\frac{\Delta \theta}{2} & \text { if } \frac{3 T}{4} \leq t \leq T
\end{array},\right.
$$


and

$$
\beta_{3}(t)= \begin{cases}\frac{\Delta \theta}{2} & \text { if } 0 \leq t \leq \frac{T}{4} \\ -\frac{4 \Delta \theta}{T} t+\frac{3 \Delta \theta}{2} & \text { if } \frac{T}{4} \leq t \leq \frac{T}{2} \\ -\frac{\Delta \theta}{2} & \text { if } \frac{T}{2} \leq t \leq \frac{3 T}{4} \\ \frac{4 \Delta \theta}{T} t-\frac{7 \Delta \theta}{2} & \text { if } \frac{3 T}{4} \leq t \leq T\end{cases}
$$

In the following, we call the "classical" Purcell stroke the one corresponding to $\Delta \theta=\frac{\pi}{3}$ and $T=4 \Delta \theta$. The time period $T$ is chosen for satisfying the constraints on the speed of deformation fixed by the optimal problem (15) (i.e., $\dot{\beta}_{i}(t) \in[-1,1], i=1,3$, for all time $t \in[0, T])$.

\subsection{Comparison of the optimal stroke with the classical Purcell stroke}

For the comparison, we take the initial position $\mathbf{x}_{2}=(0,0)$ and $\theta_{2}=0$ and the final position $\mathbf{x}_{2}=(-0.25,0)$ and $\theta_{2}=0$. We also constrain the angles $\beta_{1}(t)$ and $\beta_{3}(t)$ to vary between $-\frac{\pi}{6}$ and $\frac{\pi}{6}$ for all time $t>0$. Solving the minimum time problem with the direct method gives us a periodic solution from which we extract a candidate for the time optimal stroke. We describe this stroke in more details, and show its displacement versus the Purcell one.

Solving the optimal problem 15 we observe that the solution is periodic, as show the graphs on Fig. 3 for the angles functions $\beta_{1}, \beta_{3}$ and the $x$-displacement.
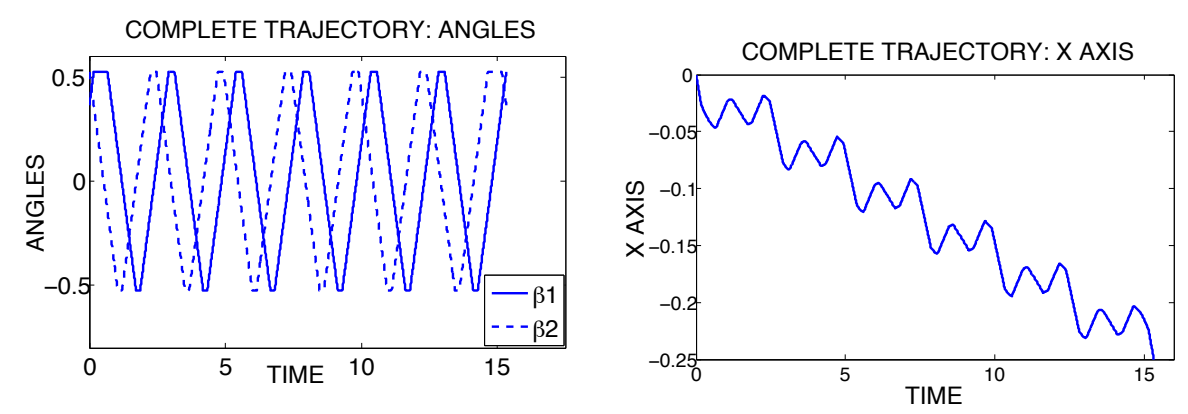

Figure 3: Angles and $x$-displacement for a whole periodic trajectory.

From the plots above it is evident that the optimal controls have a periodic structure and perform more than one period in the optimal interval of time. In order to compare the results for the displacement with the Purcell's ones, we need to select only one period (i.e. one stroke). We show on Fig. 4 the angles functions $\beta_{1}$ and $\beta_{3}$, as well as the phase portrait for both the classical Purcell stroke and our selected optimal stroke. Notice that for satisfying the constraints on the speed of deformation $\mathbf{u} \in[-1,1]$, the 
time performed by the swimmer to do the Purcell Stroke is greater than the time to do the optimal one.
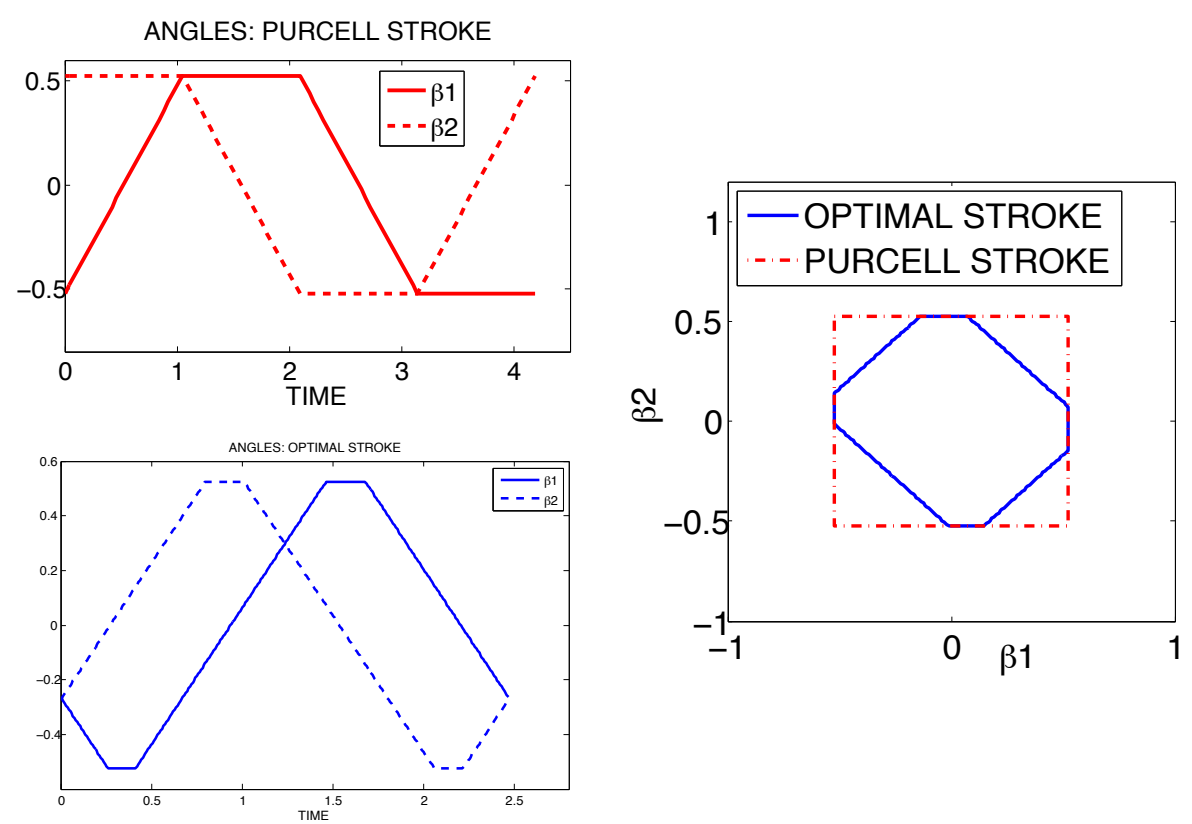

Figure 4: Angles and phase portrait - Purcell stroke and optimal stroke.

We show now the shape changes in the $(\mathrm{X}, \mathrm{Y})$ plane for the Purcell and optimal stroke. Figure 5 shows the Purcell swimmer in four different times during the classical Purcell stroke, and Fig. 6 shows the swimmer performing the selected optimal stroke.
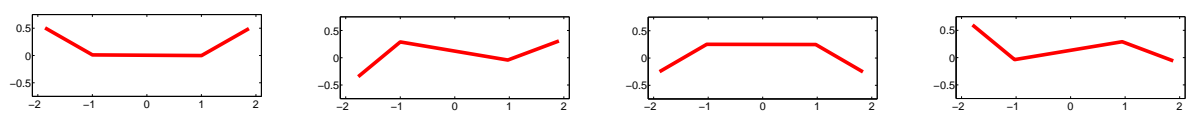

Figure 5: Shape changes for the Purcell's stroke.
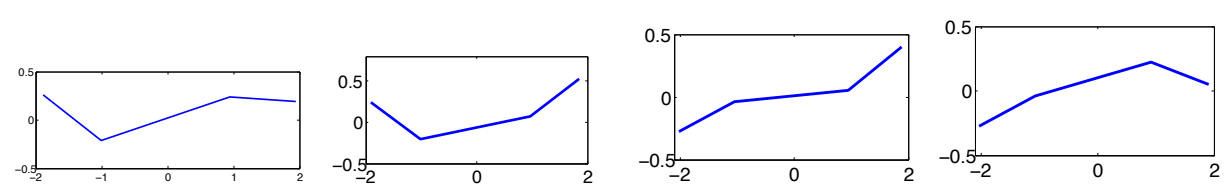

Figure 6: Shape changes for the optimal stroke.

We draw on Fig. 7 the $x$-displacement of the swimmer when it uses the classical Purcell stroke represented by the red curve and the optimal stroke depicted by the bleu 
curve. The interval of time $[0,15.3252]$ is the one given by the numeric simulation, it leads the swimmer to reach $\mathbf{x}_{2}=(-0.25,0)$ with the optimal strategy, instead by the Purcell strategy the swimmer reaches only $(\approx-0.18,0)$. We observe that our optimal stroke allows the swimmer to move further in the $x$-direction. More precisely, the optimal stroke leads a $x$-displacement close to one given by the Purcell stroke. But, the cycle of deformation of the optimal stroke is performed in less time than the Purcell one. So, for a time fixed, the optimal stroke steers the swimmer to have a greater $x$-displacement. In Fig. 7, we discern that almost 3.5 Purcell strokes are performed during the time $[0,15.3252]$, whereas there are six optimal strokes in the same time.

Remark 5.1 We see that the gap between the two curves grows with time, which confirms that the optimal stroke is better, regardless of the small difference in the initial shape of the swimmer.

Notice that the final displacement after one Purcell strokes matches the results of $[6]$.

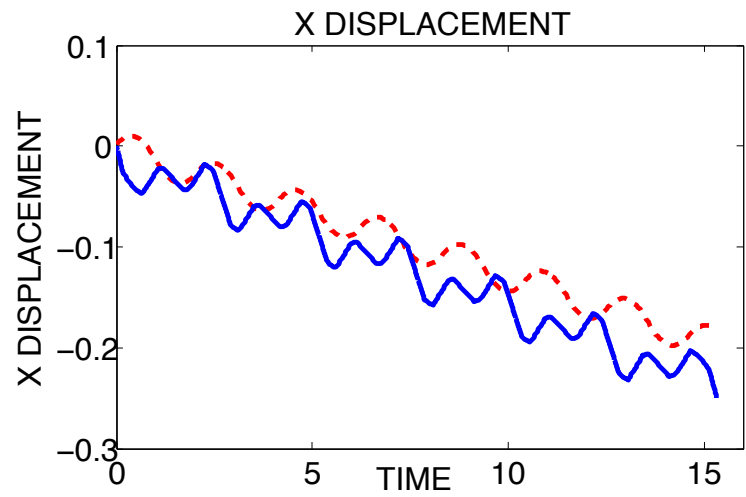

Figure 7: $\mathrm{x}$ and y displacement for one Purcell and one optimal stroke.

We study now for both strokes the x-displacement for a stroke with respect to the angular excursion, as shown on Fig. 8. In both cases, we see that a larger interval of angular excursion gives a greater displacement. Here again, it is obvious that the strokes given by our optimization strategy produce a greater speed $(x$-displacement over stroke period) than the Purcell one for any range of angular excursion. 


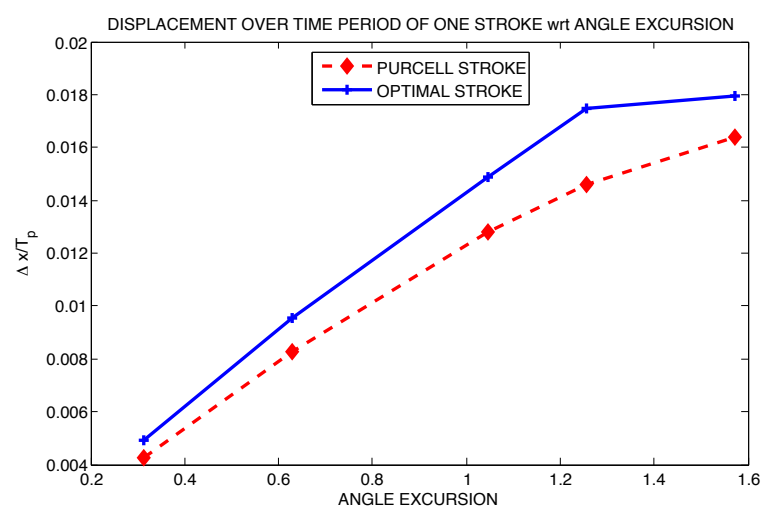

Figure 8: $x$ displacement, noted $\Delta x$, over the time period to perform a stroke, denoted by $T$, wrt angular excursion, Purcell (in red) and optimal stroke (in bleu).

\section{Conclusions}

In this paper we study the $N$-link swimmer, and use the Resistive Force Theory to derive its dynamics, as was done in [1]. In this context, we prove that for $N$ greater than 3 and for almost any $N$-uplet of sticks lengths, the swimmer is globally controllable in the whole plane. Then, we focus on finding a swimming strategy that leads the $N$-link swimmer from an fixed initial position to a given final position, in minimum time. As a consequence of the controllability result, we show that there exists a shape change function which allows to reach the final state in a minimal time. We formulate this optimal control problem and solve it with a direct approach (Bocop) for the case $N=3$ (Purcell swimmer). Without any assumption on the structure of the trajectory, we obtain a periodic solution, from which we identify an optimal stroke. Comparing this optimal stroke with the Purcell one confirms that it is better, actually giving a greater displacement speed. More precisely, the difference is due to the fact that optimal stroke is executed in less time than the Purcell one.

Current and still ongoing works include solving the optimal control problem for more complex displacements (along the y axis, rotations) and/or for different cost functions (such as energy-type). Also, noticing that the $N$-link swimmer was introduced in [1] in the perspective of approximating the motion of several living micro-organisms, an interesting extension of this model is to generalize the simulations to greater values of $N$. Of course, comparing the candidate for the optimal motion strategy with the one used by real micro-organisms could be a more tricky issue. On the other hand, another interesting direction is to study formally the existence of the periodic solution for the optimal problem. 


\section{References}

[1] F. Alouges, A. DeSimone, L. Giraldi, and M. Zoppello. Self-propulsion of slender micro-swimmers by curvature control: N-link swimmers. accepted in International Journal of Non-Linear Mechanics, 2013.

[2] F. Alouges, A. Desimone, L. Heltai, A. Lefebvre, and B. Merlet. Optimally swimming stokesian robots. DCDS-B, 2013.

[3] F. Alouges, A. DeSimone and A. Lefebvre. Optimal strokes for low Reynolds number swimmers: an example. Journal of Nonlinear Science, 2008.

[4] F. Alouges, and L. Giraldi. Enhanced controllability of low Reynolds number swimmers in the presence of a wall. Acta Applicandae Mathematicae, April 2013.

[5] P. R. Amestoy, I. S. Duff, J. Koster, and J-Y. LÉxcellent. A fully asynchronous multifrontal solver using distributed dynamic scheduling. SIAM Journal of Matrix Analysis and Applications, 23(1):15-41, 2001.

[6] L. E. Becker, S. A. Koehler, and H. A. Stone. On self-propulsion of micro-machines at low Reynolds number: Purcell's three-link swimmer. J. Fluid Mech., 2003.

[7] F. Bonnans, P. Martinon, and V. Grélard. Bocop - A collection of examples. Technical report RR-8053, INRIA, 2012.

[8] T. Chambrion and A. Munnier. Generic controllability of 3d swimmers in a perfect fluid. SIAM Journal on Control and Optimization, 50(5):2814Ü2835, 2012.

[9] J. M. Coron. Control and Nonlinearity. American Mathematical Society, 2007.

[10] B. M. Friedrich, I. H. Riedel-Kruse, J. Howard, and F. Jülicher. High-precision tracking of sperm swimming fine structure provides strong test of resistive force theory. The Journal of Experiment Biology, 2010.

[11] A. Gebremedhin, A. Pothen, and A. Walther. Exploiting sparsity in jacobian computation via coloring and automatic differentiation: a case study in a simulated moving bed process. In C. Bischof et al, editor, Lecture Notes in Computational Science and Engineering 64, pages 339-349. Springer, 2008. Proceedings of the Fifth International Conference on Automatic Differentiation (AD2008).

[12] J. Gray and J. Hancock. The propulsion of sea-urchin spermatozoa. Journal of Experimental Biology, 1955.

[13] V. Jurdjevic. Geometric control theory. Cambridge University Press., 1997.

[14] E. Lauga, T. Powers. The hydrodynamics of swimming micro-organisms. Rep. Prog. Phys. 72, 096601, 2009. 
[15] J. Lohéac, J. F. Scheid, and M. Tucsnak. Controllability and time optimal control for low Reynolds numbers swimmers. Acta Appl. Math., 123(1):175-200, 2013.

[16] R. Montgomery. A tour of subriemannian geometries, their geodesics and applications. AMS, Providence, 2002.

[17] E. Passov, and Y. Or. Dynamics of Purcell's three-link microswimmer with a passive elastic tail. European Physical Journal E 35:78, 2012.

[18] E. M. Purcell. Life at low Reynolds number. American Journel of Physics, 45:3-11, 1977.

[19] D. Tam and A. E. Hosoi. Optimal strokes patterns for purcell's three link swimmer. Physical Review Letters, 2007.

[20] G. Taylor. Analysis of the swimming of microscopic organisms. Proc. R. Soc. Lond. A, 209:447-461, 1951.

[21] E. Trelat. Contrôle optimal : théorie and applications. Vuibert, Collection Mathématiques Concrètes, 2005.

[22] A. Wächter and L.T. Biegler. On the implementation of a primal-dual interior point filter line search algorithm for large-scale nonlinear programming. Mathematical Programming, 106(1):25-57, 2006.

[23] A. Walther and A. Griewank. Getting started with Adol-c. In U. Naumann and O. Schenk, editors, Combinatorial Scientific Computing. Chapman-Hall CRC Computational Science, 2012. 